\title{
Exhibits at the Optical Convention, I926.
}

THE Optical Convention, I926, was held at the Imperial College of Science and Technology, South Kensington, during the week April I2-I7, under the presidency of the Astronomer Royal, supported by representatives of all branches of the optical industry and profession. An important part of the Convention was the exhibition of optical instruments and apparatus organised to demonstrate the advances that have been made in optics in Great Britain since I9I2, the date of the previous Convention. During the week, more than 8000 visitors, in addition to members of the Convention, attended the exhibition. Many were attracted, no doubt, by the various entertainments and performances incorporating interesting optical illusions, but many also were attracted by a desire to study the concrete evidences of recent developments and the most modern products of British opticians, many of which, as the Prime Minister pointed out during the opening ceremony, are recognised the world over as achieving the bigh water-mark of technical perfection.

Considerable development might be expected in an industry during a period of fourteen years. When it is remembered that during part of that period, development was quickened by the urgency of wartime requirements, and later by the necessity of meeting fierce competition arising from depreciated foreign exchange rates, and also of keeping pace with rapid advances in science, it can easily be imagined that the manufacturer had a wide range from which to choose his exhibits.

Optical glass, the most important of the optician's raw materials, was exhibited in the rough lump and in the form of moulded slabs, disks and prisms by Messrs. Chance Bros. and Co., Ltd., and by Messrs. Parsons' Optical Glass Co. The latter firm exhibited a disk of hard crown glass, 32 in. in diameter, suitable for use in a 3o-in. aperture telescope object-glass. Specimens of dense barium crown glass shown by Messrs. Chance Bros., illustrated the improvement in quality, particularly in regard to freedom from colour and bubbles, which has been effected in recent years in the production of this difficult glass. This firm now makes more than Ioo different types of glass suitable for optical purposes, many of which were not manufactured before the War.

Some of the uses to which these new glasses have been put were well illustrated by the extensive range of new types of photographic lenses exhibited. The Ross Xpres and the Taylor-Hobson Aviar lenses were designed and produced during the War to meet the demand for large-aperture lenses giving the high quality of definition required in air photography. Further developments in the direction of the production of rapid anastigmats were represented by Messrs. Taylor, Taylor and Hobson's $\mathrm{f} / 2 \cdot 5$ lens, suitable for reflex cameras, and also their $\mathrm{f} / 2$ anastigmat, which has a covering power of $55^{\circ}$ and represents the limit so far reached in the production of wide angle, largeaperture lenses. Another exhibit by the same firm was a series of $f / 3.5$ telephoto lenses, which marks an important advance in telephoto construction. This advance has been shared by the Teleros lenses of Messrs. Ross, Ltd,, who had on view a 40 -in. lens of that series fitted to a Ross Sports camera. Telephoto lenses of recent design were also exhibited by Messrs. J. H. Dallmeyer, Ltd., and by Messrs. Wray (Optical Works), Ltd.

The remarkable developments that have been effected in British-made surveying instruments were well shown in the various types of theodolites and levels exhibited by Messrs. C. F. Casella and Co., Ltd. Messrs. Cooke, Troughton and Simms, Ltd., Messrs. W. Ottway and Co., Ltd., and Messrs. E. R. Watts and Son, Ltd. Theseimprovements have been largely directed towards securing optical perfection, reduction in dimensions and weight, greater rigidity, more effective guarding of vulnerable parts, and the introduction of means for saving time and labour in the making of observations. In the precise levels exhibited by these firms, the telescope with the attached bubble can be tilted independently of the vertical axis, which greatly facilitates and expedites field work. Provision is also made on many of the instruments for reading the bubble, the compass bearing, and the staff, from the telescope-end position, without change of position on the part of the observer. Messrs. Cooke, Troughton and Simms and Messrs. Watts exhibited levels embodying reversible bubbles, the use of which simplifies the adjustment of the instrument. Further simplification in the use of Messrs. Watts' instruments have been effected by the introduction of their " constant" bubble, which is so constructed that changes of temperature within the range $0^{\circ}$ to $130^{\circ} \mathrm{F}$. produce no change in the length of the air bell. Thus, when the instrument is being set it is necessary to observe only one end of the bubble.

With the exception of a few standard instruments or parts of instruments, the microscope apparatus exhibited by Messrs. C. Baker, Messrs. R. and J. Beck, Ltd., Messrs. Negretti and Zambra, Messrs. Ogilvy and Co., Messrs. James Swift and Son, Ltd. and Messrs. W. Watson and Sons, Ltd., represented an entirely new series of instruments and appliances for microscopical work computed and designed since the last Convention, and including all types of microscopes and microscope accessories such as are required for students' use, for industrial processes, for scientific investigations and for all classes of laboratory and research work. The modifications that have been introduced involve many important changes in mechanical design and construction, and also improvements in optical properties. One of the most striking alterations in mechanical design is that shown in the Beck Radial microscope. The general design is that of a small optical bench so arranged that as the inclination is changed from the vertical to the horizontal, or any intermediate position, the centre of rotation remains in the optic axis. The advantage of this arrangement is specially apparent in metallurgical work when a vertical illuminator is used. The adjustment, being once set, is not altered by the inclination of the instrument or by the focussing.

Of the many important instruments exhibited by Messrs. Adam Hilger, Ltd., the most outstanding were the Hilger interferometers, including those for demonstration purposes and for the examination of prisms and lenses, and the latest model, the Universal camera lens interferometer, which permits of the examination of all ordinary photographic lenses up to a clear aperture of $5 \frac{1}{2}$ in., and from 4 in. up to about 33 in. focal length, as well as lenses of the telephoto type, process lenses, complete telescopes up to a total length of 12 in., and eyepieces. On this firm's stand were also a complete range of Hilger wave-length spectrometers; a permanent adjustment quartz spectrograph giving a spectrum from 2 Ioo $\AA$.U. to $8000 \AA$.U.; a vacuum spectrograph for the study of the furthest ultra-violet region, in which a concave diffraction grating of 2 metres radius is used; and the Hilger ultra-violet spectroscope provided with a fluorescent screen from which the wave-length may 
be directly determined. Messrs. Bellingham and Stanley, L.td., also exhibited quartz spectrographs and a range of polarimeters and spectrometers, including a compact instrument for the examination of the entire spectrum giving readings directly in wave-lengths.

The ophthalmic section of the industry was well represented by about twenty-five different exhibitors. Many new types of spectacle lenses, eyeglass mounts and spectacle frames were shown. The ophthalmic instruments on view illustrated the development of apparatus for the detection of pathological conditions of the eye, as well as those for the mechanical measurement of visual errors. Among these may be mentioned the self-registering perimeters exhibited by several firms; the photographic ophthalmoscope, exhibited by the Cambridge Instrument Co., Ltd., by means of which it is possible to obtain photographs of the fundus showing both the optic disk and the macula lutea on one plate; and the combined corneal microscope and slit lamp, exhibited by Messrs. Clement Clarke, Ltd., in which both the illuminating system and the microscope are mounted on an arc which has its centre of curvature at the focus of the illuminating beam.

It is, of course, quite impossible in an article of reasonable length to give anything approaching a full list of the various interesting pieces of apparatus on view and described in the 320-page catalogue prepared for the use of the members of the Convention. In the preceding paragraphs reference has been made to only a few of the items on the stands of a few of the trade exhibitors, and many important items in important groups of instruments have had to be omitted.

The educative value of the exhibition was enhanced by the inclusion of a section devoted to optical instruments and apparatus designed by research workers for use in scientific investigations. These included an instructive collection of apparatus exhibited by the National Physical Laboratory, Teddington; the Technical Optics Department of the Imperial College of Science and Technology, South Kensington; the Department of Applied Optics, Northampton Polytechnic Institute, London; the Refraction Hospital, London; and by other institutions and scientific workers. Mention should be made of the selenium apparatus designed by Dr. E. E. Fournier d'Albe and E. O. Symonds for registering mechanically the passage of interference fringes ; the Clarendon photoelectric photometer, exhibited by Prof. F. A. Lindemann and Dr. G. M. B. Dobson, by means of which very small areas, such as spectral lines on a photographic plate, can be measured to a high degree of accuracy; and the series of instruments exhibited by the Cambridge Instrument Co., Ltd., in which the stylus-on-celluoid method is used for recording various physical phenomena.

The historical section of the exhibition contained a valuable loan collection of more than 350 items of great historical importance, illustrating the evolution and development of various types of optical instruments and indicating the types actually made and in use at different periods. Our admiration for the many highly developed and finished products of modern science in the adjoining sections did not in any way detract from our admiration for these optical treasures of past generations; for did not each one represent a modest though none the less distinctive landmark along the path of optical progress? The magnificent and impressive display of present-day apparatus, instruments and methods which formed the Optical Convention 1926 Exhibition marks the farthest point yet reached along that path.

\section{The Intermittent Theory of Radiation.}

$\mathrm{A}^{\mathrm{N}}$ important paper on "The Intermittence of to Electric Force" was read by Sir J. J. Thomson We now know that the atom is made up of electrons and positive charges, and that the number of electrons in the atom of an element is equal to the atomic number of the element. We also know the masses of the electrons and the positive charges. If, therefore, they obeyed the ordinary laws of electrostatics, we could calculate by the laws of dynamics the behaviour of the atom. The behaviour predicted in this way is not in accord with experiment. The reason of this discrepancy is doubtless that in the atom we are concerned with effects which occur in times and in distances very much shorter than those which occurred in the experiments by which the laws of electric force were discovered or tested.

Sir Joseph directs attention to the possibility of the electric force being intermittent in its action. The intermittence is so rapid that its effects are only apparent when we are dealing with events which occur in intervals as short as those in the atom. He illustrates this point by considering the properties of gases. So long as we test these properties on an ' engineering scale' they are completely explained by supposing that the gas exerts a continuous pressure on the walls of the containing vessel and that it obeys the hydrodynamical laws for a continuous fluid. It is known, however, that the pressure is not really continuous but consists of a succession of isolated blows following each other irregularly at very small intervals of time. By the methods now available for producing very high vacua we can get the gas in a state where it is impossible to explain its be- haviour by the hypothesis of continuity. The gas has a structure in time, the fineness of which is determined by the interval between the collisions. The fineness of the structure for a given gas varies with the pressure, being coarse at low pressure and fine at high pressure.

Sir Joseph supposes that the electric field has a structure in time, so that instead of the electric force being a continuous effect consisting of a continuous flow of momentum into the body under its action, it is really discontinuous and consists of finite increments of momentum separated by finite intervals of time. This discontinuity in the nature of the force readily explains the occasional spontaneous dissociation of a system. It also gives a reasonable hypothesis to explain why a system sometimes radiates away its surplus energy as Röntgen rays.

The assumption of the intermittence of electric force leads to startling results when applied to light waves. The mathematical proofs given indicate that there can be no unlimited propagation of light waves diverging from a source. The waves must ultimately reach a region where they are reflected back.

It will thus be seen that the hypothesis demands a corpuscular theory of light. The energy of the light must be done up into batches which retain their energy undiminished as they travel out into space. This does not exclude, in fact it requires, the presence of electrical waves of Maxwell's type. We must picture the light unit as a central quantum vibrating with the period of the light and emitting electrical waves. None, however, is allowed to escape, and the energy lost by the quantum in radiation is restored to it.

$$
\text { NO. 2947, VOL. I I 7] }
$$

